\title{
Thermodynamics of computation and linear stability limits of superfluid refrigeration of a model computing array
}

\author{
Michele Sciacca $^{1,6} \quad$ Antonio Sellitto ${ }^{2} \quad$ Luca Galantucci $^{3} \quad$ David Jou $^{4,5}$ \\ ${ }^{1}$ Dipartimento di Scienze Agrarie e Forestali, Università di Palermo, Viale delle Scienze, 90128 Palermo, Italy \\ 2 Dipartimento di Ingegneria Industriale, Università di Salerno, Campus di Fisciano, 84084, Fisciano, Italy \\ ${ }^{3}$ Joint Quantum Centre (JQC) DurhamNewcastle, and School of Mathematics and Statistics, Newcastle \\ University, Newcastle upon Tyne, NE1 7RU, United Kingdom \\ ${ }^{4}$ Departament de Física, Universitat Autònoma de Barcelona, 08193 Bellaterra, Catalonia, Spain \\ ${ }^{5}$ Institut d'Estudis Catalans, Carme 47, Barcelona 08001, Catalonia, Spain \\ ${ }^{6}$ Istituto Nazionale di Alta Matematica, Roma 00185 , Italy
}

\begin{abstract}
We analyze the stability of the temperature profile of an array of computing nanodevices refrigerated by flowing superfluid helium, under variations of temperature, computing rate, and barycentric velocity of helium. It turns out that if the variation of dissipated energy per bit with respect to temperature variations is higher than some critical values, proportional to the effective thermal conductivity of the array, then the steady-state temperature profiles become unstable and refrigeration efficiency is lost. Furthermore, a restriction on the maximum rate of variation of the local computation rate is found.
\end{abstract}

Keywords: Stability analysis; Computer refrigeration; Superfluid helium; Thermodynamics of computation.

AMS Subject Classifications: 76A25, (Superfluids (classical aspects)) 80A99. (all'interno di Thermodynamics and heat transfer)

\section{Introduction}

Thermodynamics of computation [1], [2], [3], [4] is a major field of current research. On practical grounds, a relevant part of energy consumption in computers is related to refrigeration costs; indeed, heat dissipation in very miniaturized chips is one of the limiting factors in computation. On the other side, the lowest dissipation limits in computation and the concept of reversible computation [1], [2] are relevant questions in mathematical physics, as well as the linear stability analysis of the system $[5,6]$.

In this paper we combine some concepts of thermodynamics of computation with the stability of computer refrigeration in the particular case of superfluid refrigeration. The motivation is quantum computation, which must be carried out at very low temperature, in order to maximize as much as possible the duration of quantum coherent states of the system [7], [8], [9], [10]. Amongst the refrigeration techniques, the use of superfluid helium is one of the most practical possibilities [11], [12], [13], [14], as also confirmed by its use as refrigerator at CERN

\footnotetext{
${ }^{0}$ E-mail addresses: michele.sciacca@unipa.it (M. Sciacca), ant.sellitto@gmail.com (A. Sellitto), luca.galantucci@newcastle.ac.uk (L. Galantucci), david.jou@uab.cat (D. Jou).
} 


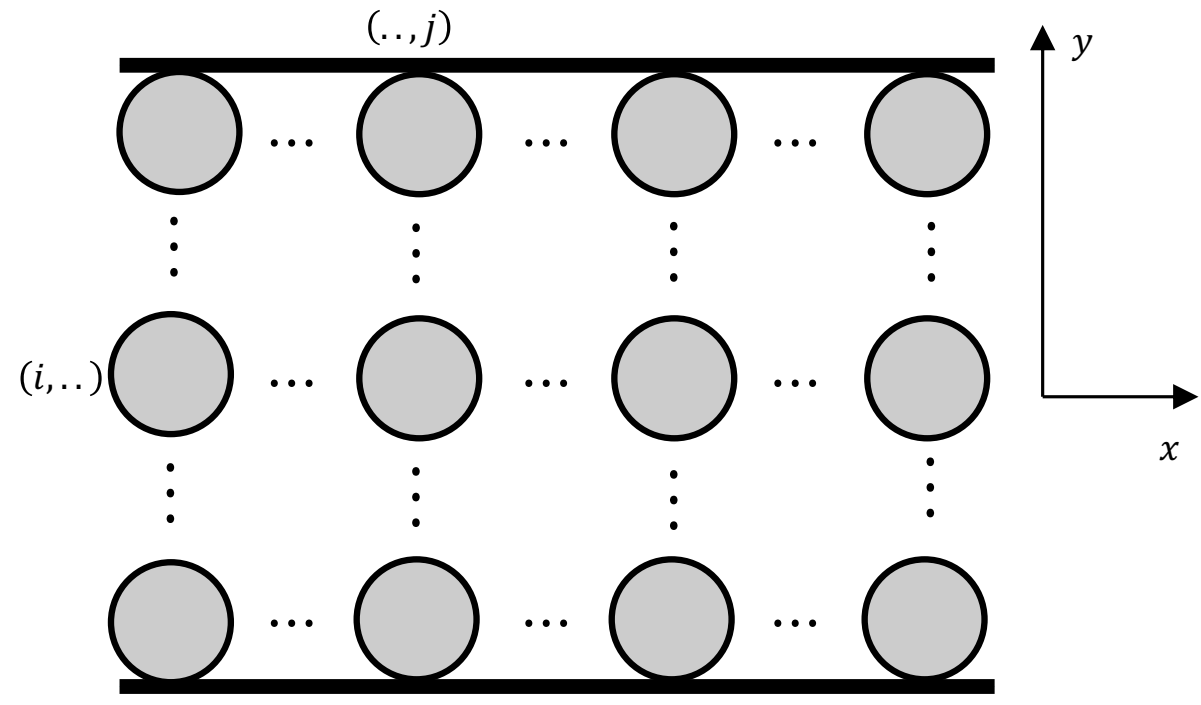

Figure 1: An overhead view of the hot-dissipating cylinders inside a channel with rectangular cross-section, filled with refrigerating superfluid helium. The heat flows along $x$-direction whereas $y$ and $z$ refers to the transversal directions, with $-a / 2 \leq y \leq a / 2$ and $0 \leq z \leq b, a$ and $b$ being the width and thickness of the channel, which is assumed to be very long in the flow direction (along the $x$ axis).

and its ability to flow in very narrow channels. In our previous papers [15], [16], [17] we have modeled the system to be refrigerated as a regular array of dissipating nanocylinders, each of them representing a set of several chips. To optimize the operation of the system, a regular, stable refrigeration is needed. Thus, the analysis of the stability of the temperature profile is a practically relevant topic in the search for optimal cryogenic stategies of such arrays. In the present paper we complement previous analyses [15], [16], [17] by considering the stability of the temperature profile.

In our simplified model, we considered that the nanocylinder in the position $(i, j)$ of the array (with $i$ and $j$ natural numbers, respectively, indicating the row and the column of the corresponding nanocylinder in the array, see Fig. 1) was dissipating a heat $\dot{Q}_{i j}$ per unit time. We assumed that this heat was related to the operations carried out in the devices, in such a way that one may indicatively consider $\dot{Q}_{i j}=\dot{n}_{i j} T_{i j} s_{i j}^{\prime}(T)$, with $\dot{n}_{i j}$ being the computation rate (number of bits processed per second), $T_{i j}$ the temperature of the $(i, j)$ device and $s_{i j}^{\prime}(T)$ the entropy produced for each bit being processed. This is the subject of many analyses of thermodynamic limits of computation [18].

Nowadays, the most efficient computers dissipate some $10^{-17} \mathrm{~J}$ per elementary operation (the processing of one bit) at room temperature, whereas the classical theoretical limit would be of the order of $K_{B} T \ln 2$, which is of the order of $10^{-21} \mathrm{~J}$ per elementary operation at room temperature. The difference between actual dissipation and theoretical minimum dissipation is related to dissipative effects in electron motion in a transistor [19], [20]. The number of transistors per unit area may be very high, because the progress in miniaturization has led to 
a dramatic increase of the density of transistors per unit area of the device.

In our previous analyses we considered $\dot{Q}_{i j}$ constant in time. Of course, this is not totally realistic, because $\dot{n}_{i j}$ is expected to change in time according to the computation needs of the program. Since this rate will change in time, we will consider its variations as a perturbation. Furthermore, the temperature profile itself may have some spontaneous perturbations, and the local velocity $\mathbf{v}$ of the superfluid helium may also fluctuate. Thus, we will study the stability of the temperature profile subject to these three sources of perturbation.

In Section 2 we present the mathematical model used in this paper and we briefly review the results of our previous papers on this topic and we state new further mathematical results; in Section 3 we study the linear stability of the temperature profile and the bounds on processing rate (dissipated heat) which may be dealt with by using helium cryogenics. Section 4 is devoted to the discussion of the main results of the paper.

\section{The mathematical model}

In this section we derive the effective thermal resistance of liquid helium through an array of parallel cylinders between two parallel plates orthogonal to the cylinders. Though the main results $(2.21),(2.22)$ and (2.23) were derived in Ref. [16] here we clarify and generalize some mathematical aspects of the previous derivation. Note that heat transport in superfluid helium is not described by Fourier's law, but by more complicated equations, which are able to cope with the complexities of this system.

We aim to consider heat removal from the system by means of superfluid helium with and without net mass flow. For this reason we consider the basic equations for the specific volume (volume per unit mass) $V=\rho^{-1}, \rho$ being the total mass density, the velocity $\mathbf{v}$, the specific internal energy $\epsilon$ and heat flux q which are [21], [22], [23], [24], [25]

$$
\begin{aligned}
& \rho \dot{V}-\nabla \cdot \mathbf{v}=0 \\
& \rho \dot{\epsilon}+\nabla \cdot \mathbf{q}+p \nabla \cdot \mathbf{v}=\sigma^{\epsilon} \\
& \rho \dot{\mathbf{v}}+\nabla p-\eta \nabla^{2}\left(\mathbf{v}+\frac{\mathbf{q}}{S T}\right)=\mathbf{0} \\
& \dot{\mathbf{q}}+\frac{\lambda_{1}}{\tau} \nabla T-\frac{\eta \lambda_{1}}{\tau S} \nabla^{2}\left(\mathbf{v}+\frac{\mathbf{q}}{S T}\right)=\sigma^{\mathbf{q}}
\end{aligned}
$$

where $S$ is the entropy per unit volume, $p$ pressure, $T$ temperature, $\tau$ the relaxation time of $\mathbf{q}$, $\lambda_{1}$ and $\eta$ can be interpreted as the thermal conductivity and the shear viscosity respectively, when applied to a classical fluid [23], the ratio $\lambda_{1} / \tau$ is related to the second-sound speed. The rigth-hand side $\sigma^{\mathbf{q}}$ of equation (2.4) is the production term, which in the laminar regime is

$\sigma^{\mathbf{q}}=\frac{1}{\tau} \mathbf{q}$ and that in the presence of quantized vortices becomes $\sigma^{\mathbf{q}}=\frac{1}{\tau} \mathbf{q}+K L \mathbf{q}$, with $L$ being the vortex length density (giving the length of of quantized vortices per unit volume) and $K$ is a coefficient proportional to the quantum of circulation $\kappa$. In Refs. [26] and [24] we have evaluated how much the presence of quantized vortices reduces the efficiency of the refrigeration by means of superfluid helium and in Refs. [27, 28] how the profile of the heat flux (and the normal component) is modificed by their contribution.

The continuity equation (2.1) has been written in terms of the specific volume $V$ instead of the usual density $\rho$ for future purposes. Since we want to avoid quantum turbulence we will take $L=0$ in $\sigma^{\mathbf{q}}$; otherwise, an additional evolution equation for $L$ and the term $K L \mathbf{q}$ 
in $\sigma^{\mathbf{q}}$ should be included. Eq. (2.4) replaces the classical Fourier's law in the description of heat transfer in HeII. Thus, the classical results for heat transfer in arrays described by Fourier's law must be modified in order to account for heat transport in the system we are considering. The source term $\sigma^{\epsilon}$ in Eq. (2.2) corresponds to the supplies of internal energy acting on the system. In our case they represent the heat-dissipating nanodevices whose heat we want to remove from the system, namely, the product of the number of bits processed per unit time times the energy dissipated per bit. For instance, at a processing rate of $4 \mathrm{GHz}$ and a dissipated energy of $10^{-15} \mathrm{~J}$ per bit processed, a representative value of the heat dissipated in every nanocylinder of the array would be of the order of $4 \cdot 10^{-4} \mathrm{~W}$, at room temperature. This value could be much reduced at low temperature and in quantum computation.

In Eqs. (2.1)-(2.4) the upper dot stands for material derivatives, i.e. $\frac{\partial}{\partial t}+(\mathbf{v} \cdot \nabla)$. In Eq. (2.2), $\sigma^{\epsilon}$ is given by the particular system we want to refrigerate, which will be related to the computation rate below, and the main physical challenge is to obtain $\mathbf{q}$, which is the heat transported inside the system by conduction, or by internal convection.

Let's briefly recall what we have found in our previous studies [16], [26]. First, let us consider the flow inside the channel with rectangular cross-section ( $x$ being the coordinate along the channel, and $y$ and $z$ the transversal coordinates). The hypothesis of long channel (as compared to its width $a$ along $y$ and thickness $b$ along $z$ ) and laminar regime of the flow allows the fields $\mathbf{v}$ and $\mathbf{q}$ to be $x$-independent. We also assume that $\sigma^{\mathbf{q}}=0$ in Eq. (2.4) because $L=0$ and $\mathbf{q} / \tau$ is negligible (since it is experimentally found to be very small because of the high value of $\tau$ ).

For the boundary conditions, in the two-fluid model it is assumed that the normal component is a viscous fluid and that the superfluid component is an inviscid fluid, which suggests to set $\mathbf{u}_{\mid \partial \sigma}^{(n)}=0$ and slip condition for $\mathbf{u}^{(s)}$, with $\mathbf{u}^{(n)}$ and $\mathbf{u}^{(s)}$ being the normal and superfluid velocity, respectively, and with $\sigma$ the transversal section of the channel and $\partial \sigma$ its countour. In the context of the one-fluid extended model, superfluid helium is described by equations (2.1) $-(2.4)$ with the issue on the boundary conditions for all the fields involved in the model (2.1)-(2.4) still open. Our statement is confirmed from some recent experimental studies performed by the La Mantia's group (Prague), who have investigated the interaction between particles and quantum turbulence in the proximity of the heater in counterflow experiments in superfluid helium. They have found an unexpected different behaviour in the proximity of the heater than in the bulk: vortex line density is two order of magnitude higher near the heater than that in the bulk. Thus, according to the rule $L^{1 / 2} \propto v_{n s} \propto q$ (where $v_{n s}$ is the modulus of the average relative velocity between the normal component and the superfluid component, and $q$ is th modulus of the heat flux), this would mean that vortex line density $L$ near the heater is higher because of the higher value of the counterflow velocity $v_{n s}$ (or equivalently the heat flux $q$ ). However, it is expected that the heat flux is the same along the mean flux direction, contraddicting the rule $L^{1 / 2} \propto q$. Thus, more efforts for understanding the contribution of the boundary conditions are worth of future consideration both from theoretical and experimental point of view. This uncertainty will be reflected to all the fields involved in Eqs. (2.1)-(2.4). Regarding the velocity field, a choice may be to set $\mathbf{v}_{\mid \partial \sigma}=0$ because of the small viscosity $\eta$ in superfluids. The boundary condition of the heat flux $\mathbf{q}$ is instead more arduous to choose. In phonon hydrodynamics (when the mean free path of the phonons is smaller than the smallest size of the system) it is usually set $\mathbf{q}_{\mid \partial \sigma}=\mathbf{0}$, as made in Refs. $[24,16]$, whereas in dilute phonon flow (namely, when the mean free path of the phonons is of the same order of the size 
of the system) the usual constant boundary condition is $\mathbf{q}_{\mid \partial \sigma}=C \ell \frac{\partial \mathbf{q}}{\partial \mathbf{n}} \mid \partial \sigma$, with $C$ and $\ell$ being a numerical constant of the order of the unity and $\ell$ the mean free-path of the heat-carriers $[29,26]$. Here, for the sake of simplicity and lack of suitable information, we choose $\mathbf{q}_{\mid \partial \sigma}=0$ as in phonon hydrodynamics. Thus, we can assume, as in Refs $[24,16]$, that:

$$
\mathbf{v}_{\mid \partial \sigma}=\mathbf{q}_{\mid \partial \sigma}=0 .
$$

We advise the reader to note that conditions (2.5) refer to the one-fluid model and that it has not to be extended to the two-fluid model, where it would lead to the contradictory condition $\mathbf{u}_{\mid \partial \sigma}^{(s)}=0$. The reader is aware that the question of the boundary conditions on superfluid helium is still open and it has not been well-considered neither theoretically nor experimentally.

We want to point out that our results are still valid if we choose the less restrictive boundary conditions:

$$
\left(\mathbf{v}+\frac{1}{S T} \mathbf{q}\right)_{\mid \partial \sigma}=0
$$

instead of (2.5).

Thus, in [26] we found that the stationary solution of equations (2.1)-(2.4) with the boundary conditions (2.5), (or alternative (2.6)) $\sigma^{\mathbf{q}}=\mathbf{0}, \sigma^{\epsilon}=0$ and $\nabla p$ constant is as follows:

$$
v(y, z)+\frac{1}{S T} q(y, z)=-\frac{4 b^{2}}{\pi^{3}} \frac{1}{\eta} \sum_{n, o d d}^{\infty} \frac{1}{n^{3}}\left[1-\frac{\cosh \left(n \pi \frac{y}{b}\right)}{\cosh \left(n \pi \frac{a}{2 b}\right)}\right] \sin \left(n \pi \frac{z}{b}\right) \nabla p .
$$

This result corresponds to the steady-state solution of equation (2.3) which has the form of Navier-Stokes equation for the variable $\mathbf{u}^{(n)}:=\mathbf{v}+\frac{1}{S T} \mathbf{q}$. The field $\mathbf{u}^{(n)}$ naturally arises from the theory of one-fluid extended model (based on the Extended Thermodynamics) and it has the dimension of velocity. It was identified as the velocity of the normal component in the two-fluid extended model, where the word extended refers to the theory of the Extended Thermodynamics [25]. From the equations of the one-fluid model (2.1)-(2.4), an evolution equation for $\mathbf{u}^{(n)}$ can be obtained (see Ref. [25] for more details), which, neglecting the bulk viscosity, is:

$$
\dot{\mathbf{u}}^{(n)}=-\frac{1}{\rho_{n}} \nabla p_{n}-\frac{\rho_{s}}{\rho_{n}} s \nabla T+\frac{\eta}{\rho_{n}} \nabla^{2} \mathbf{u}^{(n)}
$$

where $\dot{\mathbf{u}}^{(n)}=\frac{\partial}{\partial t} \mathbf{u}^{(n)}+\left(\mathbf{u}^{(n)} \cdot \nabla\right) \mathbf{u}^{(n)}$ and $\nabla p_{n}=\nabla p-\rho_{s}\left(\left(\mathbf{u}^{(n)}-\mathbf{u}^{(s)}\right) \cdot \nabla\right) \mathbf{u}^{(n)}$. Assuming that $\sigma^{\epsilon}=0$ and $\dot{V}=\dot{\epsilon}=0$ in Eqs. (2.1)-(2.4), then we recover the further condition $\nabla \cdot \mathbf{u}^{(n)}=0$. Thus, reading the term $\nabla p_{n}+\rho_{s} s \nabla T$ in (2.8) as the gradient of a net pressure $P$, equation (2.8) formally becomes the Navier-Stokes equation with $\nabla \cdot \mathbf{u}^{(n)}=0$.

In Ref [30] the stability of the laminar flow of a classical fluid in a rectangular duct has been investigated in terms of the Reynolds number $R e$ and the aspect ratio $\varphi=\frac{a}{b}$ of the rectangular cross-section ( $a$ and $b$ being the two sides of the rectangle). More in details, it is assumed that the total velocity and pressure fields are defined by:

$$
\begin{aligned}
& \mathbf{u}(\mathbf{x}, t)=\mathbf{u}_{0}(\mathbf{x})+\hat{\mathbf{u}}(y, z) \exp (\mathrm{i} \alpha(x-c t)) \\
& P(\mathbf{x}, t)=P_{0}(\mathbf{x})+\hat{P}(y, z) \exp (\mathrm{i} \alpha(x-c t))
\end{aligned}
$$


where $\mathbf{u}_{0}(\mathbf{x})$ and $P_{0}(\mathbf{x})$ are, respectively, the stationary values of the velocity and pressure, $\hat{\mathbf{u}}(y, z)$ and $\hat{P}(y, z)$, instead, are the small disturbance of the two corresponding fields ( $x$ is the direction of the duct, $y$ and $z$ are the coordinates in the orthogonal direction), $\alpha$ is the wavenumber along the $x$-axis and $c=c_{r}+\mathrm{i} c_{i}$ is constant, which may depend on $\alpha$. The authors analyzed the basic flow with respect to four modes for Reynolds numbers up to 50000 and wavenumbers below 1.1. The results of the linear stability is that the flow is stable for small aspect ratio $\varphi \lesssim 3.2$ while for $\varphi \gtrsim 3.2$ the stability of the flow depends on the Reynolds number $R e$ and the wavenumbers $\alpha$ by means of the thresholds $R e_{c}$ and $\alpha_{c}$. The instability arises from the first two modes that they find: the mode I shows lower $R e_{c}$ and higher $\alpha_{c}$ than the ones in the mode II. For instance, for the aspect ratio $\varphi=6$ mode I shows instability for values higher than $R e_{c}=8200$ and $\alpha_{c}=0.94$, whereas mode II shows instability for values higher than $R e_{c}=31500$ and $\alpha_{c}=0.69$. In the case of $\varphi=8$, they find $R e_{c}=6800$ and $\alpha_{c}=0.98$ for mode I, and $R e_{c}=11000$ and $\alpha_{c}=0.89$ for mode II. Other exstimations for mode I are: a) aspect ratio $\varphi=3.5$ and $R e_{c}=36600$ and $\alpha_{c}=0.71$, or $\varphi=4$ and $R e_{c}=18400$ and $\alpha_{c}=0.71$, or $\varphi=5$ and $R e_{c}=10400$ and $\alpha_{c}=0.91$, or $\varphi=\infty$ (plane Poiseuille flow) and $R e_{c}=5772$ and $\alpha_{c}=1.02$ [30].

These results can be applied to the velocity of the extended normal fluid field $\mathbf{u}^{(n)}$ and pressure $P=p_{n}+\rho s T$ under the hypothesis mentioned below equation (2.8). Of course, these results are strongly related to our hypothesis on the boundary conditions (2.5) (or alternatively (2.6)). However, since a more general expression for the boundary conditions does not preclude our choice, which we think to be physically realistic, we claim that the above thresholds for Reynolds number and the wavenumber will hold for the instability of $\mathbf{u}^{(n)}$. On the other hand, we cannot make strong statements with respect of the stability of the flow. The classical Reynolds number $R e$, in terms of $\mathbf{u}^{(n)}$, takes the form

$$
R e_{g}:=\left(v+\frac{q}{S T}\right) \frac{\rho a}{\eta}
$$

with the appearance of the average speed $v$ and the average heat flow $q$ along the channel. Thus, expression (2.11) defines a generalized Reynolds number and the results from [30] can be read in terms of $R e_{g}$ instead of $R e$. Thus we can assert the following Remark.

REMARK 2.1 The stationary solution (2.7) is linear unstable for $R e_{g}>R e_{g c}$, where $R e_{g c}$ is the threshold of $R e_{g}$, and in particular for $\varphi \rightarrow \infty$ then $R e_{g c}=5772$, as found in Ref. [30].

In Refs. [16], [26] we have evaluated the total flow of the field (2.7) across a transversal section of the rectangular channel and we have found

$$
\nabla T=-\frac{12}{S b^{3} a} \eta\left(a b \bar{v}+\frac{1}{S T} \dot{Q}\right)\left[1-\sum_{n, o d d}^{\infty} \frac{1}{n^{5}} \frac{192}{\pi^{5}} \frac{b}{a} \tanh \left(n \pi \frac{a}{2 b}\right)\right]^{-1},
$$

where $\bar{v}$ is the mean value of the barycentric velocity, and $\dot{Q}$ is the total heat current accross the transverse area. For $\varphi=a / b \gg 1$, Eq. (2.12) becomes

$$
\nabla T=-\frac{12}{S b^{3} a} \eta\left(a b \bar{v}+\frac{1}{S T} \dot{Q}\right)\left[1-\frac{0.63}{\varphi}\right]^{-1}
$$

Now we distinguish two cases: a) the counterflow situation $(\bar{v}=0)$, i.e. absence of net mass flow, and $\mathrm{b})$ the coflow situation $(\bar{v} \neq 0)$, i.e. with net mass flow. 
REMARK 2.2 One difference between our work and classical hydrodynamics is the appearance of $v+\frac{q}{S T}$ instead of $v$ in equation (2.3) and in the Reynolds number (2.11). Note that if we write $R e_{g}=R e+R e_{g}^{q}=\left(\frac{v \rho a}{\eta}\right)+\left(\frac{q}{S T} \frac{\rho a}{\eta}\right)$, with $R e=\left(\frac{v \rho a}{\eta}\right)$ the classical Reynolds number and $R e_{g}^{q}=\left(\frac{q}{S T} \frac{\rho a}{\eta}\right)$, we see that turbulence can be established because of $R e$ (velocity $v$ ) or $R e_{g}^{q}$ (heat flux $q$ ). It is unclear how this kind of turbulence of $\mathbf{q}$ may be connected to quantum turbulence, which refers to the appearance of quantized vortices for quantum thermal Reynolds number $R e_{q}=\frac{q}{S T} \frac{a}{\kappa}$, where $\kappa$ is the the quantum of circulation $(h / m)$ which has the same dimensions (i.e. length ${ }^{2} /$ time) than $\eta / \rho$. Quantum turbulence in counterflow experiments appears approximately for $R e_{q}>210$ at $T=1.7 \mathrm{~K}$, namely for $R e_{g}^{q}=\frac{q a}{S T} \frac{\rho}{\eta}>210 \frac{\kappa \rho}{\eta}=2350$ (because $\eta / \rho=8.997 \cdot 10^{-9} \mathrm{~m}^{2} / \mathrm{s}$ at $T=1.7 \mathrm{~K}$ ) [31]. Thus, quantum turbulence will appear for lower values of $R e_{g}^{q}$ than classical heat turbulence, because the latter appears, as mentioned above, for $\operatorname{Re}_{g}^{q}$ higher than 5772 , whereas quantum turbulence at $T=1.7 \mathrm{~K}$ appears for $R e_{g}^{q}$ higher than 2350. It follows from here that quantum turbulence puts a more restrictive condition than classical turbulence on the transport problem we are considering.

Definition 2.1 The thermal resistance of the channel is defined as

$$
R_{\mathrm{eff}}^{\mathrm{ch}}=\left(K_{\mathrm{eff}}^{\mathrm{ch}}\right)^{-1}=-\frac{A}{\dot{Q}} \nabla T
$$

where $A$ is the transversal area. This is analogous to the electrical resistance according to the Ohm's law $\Delta V=R I$, with $R$ the resistance and $I$ the current intensity (total electric charge crossing the conductor per unit time) and it is the reciprocal of the conductance. Therefore, according to Eqs. (2.13) and (2.14), $R_{\text {eff }}^{\text {ch }}$ is given by

$$
R_{\mathrm{eff}}^{\mathrm{ch}}=\frac{12 \eta}{b^{2} S} \frac{1}{\left[1-\frac{0.63}{\varphi}\right]}\left(\frac{a b \bar{v}}{\dot{Q}}+\frac{1}{S T}\right),
$$

which in counterflow (approximately $\bar{v}=0$ ) becomes

$$
R_{\mathrm{eff}}^{\mathrm{ch}}=\frac{12 \eta}{b^{2} S^{2} T} \frac{1}{\left[1-\frac{0.63}{\varphi}\right]} .
$$

REMARK 2.3 Note that in coflow $\nabla T$ is higher when the mean mass and heat flux point towards the same direction. Instead, $\nabla T=0$, namely $T$ constant, for $\bar{v}=-\frac{1}{a b S T} \dot{Q}$.

In [16] we have calculated the temperature drop through an array of infinitely long cylinders in the absence of mass flow $\bar{v}=0$. The direction of the applied heat flux was assumed to be orthogonal to the cylinders of the arrays. For an array of a lattice of $M$ columns of $N+1$ cylinders (of radius $R$ and separation $2 c$ between the center of the neighboring cylinders) we found

$$
\begin{gathered}
(\Delta T)_{c y l} \equiv \frac{3 M \eta}{2 b c^{2} S^{2} T} \frac{\dot{Q}}{N}\left\{2(1-\phi)+\frac{\phi^{2}}{\left(1-\phi^{2}\right)^{5 / 2}}\right. \\
\left.\left[\frac{3 \pi}{2}+\frac{\left(2+\phi^{2}\right)}{\phi} \sqrt{1-\phi^{2}}+3 \arctan \left(\frac{\phi}{\sqrt{1-\phi^{2}}}\right)\right]\right\}
\end{gathered}
$$


where $\phi=R / c$. This leads to the following effective thermal conductivity

$$
\begin{aligned}
& K_{\mathrm{eff}}^{\mathrm{cyl}}=\frac{2 S^{2} T c^{2}}{3 \eta(1-\phi)}\left\{2(1-\phi)+\frac{\phi^{2}}{\left(1-\phi^{2}\right)^{5 / 2}}\right. \\
& \left.\left[\frac{3 \pi}{2}+\frac{\left(2+\phi^{2}\right)}{\phi} \sqrt{1-\phi^{2}}+3 \arctan \left(\frac{\phi}{\sqrt{1-\phi^{2}}}\right)\right]\right\}^{-1},
\end{aligned}
$$

where the total transversal width is $a=2 N(c-R)=2 c N(1-\phi)$ and the total length along the channel is $l=2 c M[16]$. Note indeed that $2(c-R)$ is the width of the gap between neighboring cylinders.

Equation (2.18) can be written in terms of the thermal resistance as

$$
\begin{aligned}
& R_{\mathrm{eff}}^{\mathrm{cyl}}=\frac{3 \eta(1-\phi)}{2 S^{2} T c^{2}}\left\{2(1-\phi)+\frac{\phi^{2}}{\left(1-\phi^{2}\right)^{5 / 2}}\right. \\
& \left.\left[\frac{3 \pi}{2}+\frac{\left(2+\phi^{2}\right)}{\phi} \sqrt{1-\phi^{2}}+3 \arctan \left(\frac{\phi}{\sqrt{1-\phi^{2}}}\right)\right]\right\} .
\end{aligned}
$$

Postulate 2.1 The total resistance through the columns of cylinders inside a plane channel is

$$
R_{\mathrm{eff}}^{\mathrm{tot}}=R_{\mathrm{eff}}^{\mathrm{cyl}}+R_{\mathrm{eff}}^{\mathrm{ch}} .
$$

In fact, this could fail if the thickness of the channel becomes comparable (or smaller than) the average separation between cylinders. Indeed, equation (2.19) has been computed assuming a velocity profile independent of the position along the cylinders (i.e. independent of the axis). Instead, equation (2.1) considers a $z$-dependent velocity profile. Studying the exact form of the resistance in these conditions would be very complicated, and we will not consider it.

Let's now consider instead of $M$ arrays of infinitely long cylinders, $M$ array of cylinders of length $b$ between two parallel walls separated by a distance $b$. The effective total width for fluids transport will be $a=N 2(c-R)=N 2 c(1-\phi)$ and legth $l=M 2 c$, where $\phi=R / c$, then in [16] and [17] we have found the total effective thermal conductivity $K_{\text {eff }}$ for such an array of cylinders inside the mentioned rectangular channel, which can be written in terms of the thermal resistance $R_{\text {eff }}^{\text {tot }}$

$$
\begin{aligned}
& R_{\text {eff }}^{\mathrm{tot}}=\frac{12 \eta}{b^{2} S^{2} T} \frac{1}{\left[1-\frac{0.63}{\varphi}\right]}+\frac{3 \eta}{2 S^{2} T c^{2}}(1-\phi) . \\
& \left\{2(1-\phi)+\frac{\phi^{2}}{\left(1-\phi^{2}\right)^{5 / 2}}\left[\frac{3 \pi}{2}+\frac{\left(2+\phi^{2}\right)}{\phi} \sqrt{1-\phi^{2}}+3 \arctan \left(\frac{\phi}{\sqrt{1-\phi^{2}}}\right)\right]\right\}
\end{aligned}
$$

where $\varphi$ is the aspect ratio $\varphi=a / b=2 c N(1-\phi) / b$.

In Fig. 2 we have plotted the quantity $\frac{S^{2} T R_{\text {eff }}^{\text {tot }}}{\eta}$ for 100 columns of 100 hot cylinders inside a thermal counterflow $(\bar{v}=0)$ channel with sizes $b=100 \mathrm{~nm}$ and three values of $c$, namely $c=100 \mathrm{~nm}, c=150 \mathrm{~nm}$ and $c=200 \mathrm{~nm}$, as function of $\phi=R / c$, i.e. for different values of cylinders radii. Note that the mentioned ratio depends only on the geometry of the array (namely, on $N, M, c, R$ and $b$ ), whereas the influence of helium prperties and of temperature appears through $\eta, S$ and $T$. Thus, for instance, the value $\phi=0.3$ in Fig. 2 (blue line) would refer to a lattice of cylinders with $R=30 \mathrm{~nm}$ separated by a gap of $40 \mathrm{~nm}$. 


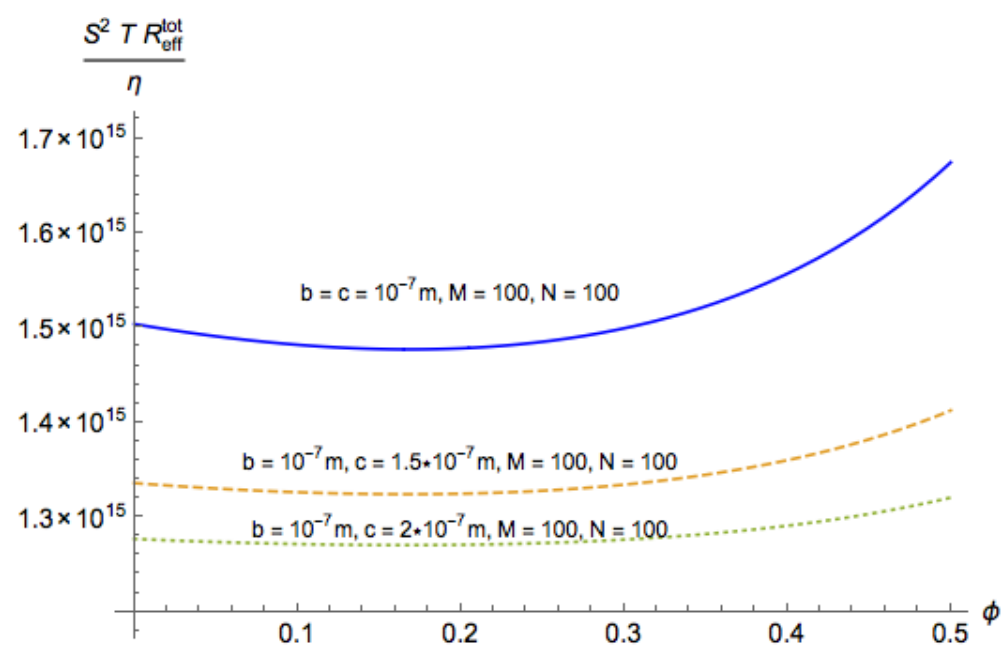

Figure 2: [color online] The total normalized thermal resistance $\frac{S^{2} T R_{\text {eff }}^{\text {tot }}}{\eta}$, with $R_{\text {eff }}^{\text {tot }}$ given by (2.21), is plotted versus the aspect ratio $\phi$ for $M=100$ columns of $N=100$ hot cylinders inside a thermal counterflow $(\bar{v}=0)$ channel with height $b=100 \mathrm{~nm}$. The three plots in figure refer to three values of $c: c=100 \mathrm{~nm}, l=2 c M=2 \cdot 10^{-5} \mathrm{~m}$ and $\varphi=a / b=2 c N(1-\phi) / b=$ $2 \cdot 10^{2}(1-\phi)$ (blue line); $c=150 \mathrm{~nm}$ (dashed yellow line), and $l=2 c M=3 \cdot 10^{-5} \mathrm{~m}$ and $\varphi=a / b=2 c N(1-\phi) / b=3 \cdot 10^{2}(1-\phi) ;$ and $c=200 \mathrm{~nm}, l=2 c M=4 \cdot 10^{-5} \mathrm{~m}$ and $\varphi=a / b=2 c N(1-\phi) / b=4 \cdot 10^{2}(1-\phi)$ (green dotted line).

For such a system, narrow separation between cylinders means $1-\phi \ll 1 \Leftrightarrow \phi \rightarrow 1$, i.e., the spacing between the cylinders is much smaller than their cross-sectional dimensions; wide separation between cylinders means $\phi \ll 1 \Leftrightarrow \phi \rightarrow 0$, i.e., the spacing between the cylinders is much bigger than their radius $R$. This means that for transport through an array of cylindrical obstacles with narrow separation between cylinders [16], i.e. for $1-\phi \ll 1 \Leftrightarrow \phi \rightarrow 1,(2.21)$ reduces to

$$
R_{\mathrm{eff}}^{\mathrm{tot}}=b(1-\phi) \frac{\eta}{S^{2} T}\left\{\frac{12}{b^{3}(1-\phi)\left(1-\frac{0.63}{\varphi}\right)}+\frac{9 \pi}{2 b c^{2}}\left[\frac{\phi^{2}}{\left(1-\phi^{2}\right)^{5 / 2}}\right]\right\}
$$

and for wide separation between cylinders, i.e. for $\phi \ll 1 \Leftrightarrow \phi \rightarrow 0,(2.21)$ reduces to

$$
R_{\mathrm{eff}}^{\mathrm{tot}}=b(1-\phi) \frac{\eta}{S^{2} T}\left\{\frac{12}{b^{3}(1-\phi)\left(1-\frac{0.63}{\varphi}\right)}+\frac{3}{b c^{2}}\left[\frac{1}{\left(1-\phi^{2}\right)^{2}}\right]\right\},
$$

where $\phi=R / c$.

\section{Stability of the solution for the temperature profile}

In previous papers [16], [17] we considered two steady-state cryogenic situations removing heat dissipated by the mentioned array. Here, we analyze the stability of such situations.

In Ref. [16] it was assumed that heat was flowing by pure counterflow: because of the symmetry of the system, it was flowing from $i=0$ to $i=N$ and to $i=-N$ (the upper and lower boundaries were assumed to be insulating in our particular example). We obtained the 
steady state temperature $T_{i}$ of the He II at the several positions $i \in[-N, N]$ along the $x$-axis (in fact, He II is considered as a continuous system, and its temperature is defined over the whole continuous zone it is occupying, but for our purpose knowing the temperature at the discrete set of positions of the cylinders is sufficient to characterize the state of the system for practical needs).

In Ref. [17] it was assumed the presence of a forced helium convection with volume flow $\dot{Q}_{v} \equiv A \mathbf{v}$ (with $A=a b$ the transversal area of the channel) crossing the system from $i=-N$ to $i=N$. In continuous terms, the equation of enthalpy $h=\epsilon+p V$, obtained combining equations (2.1) and (2.2), becomes

$$
\rho \frac{\partial}{\partial t} h+\rho(\mathbf{v} \cdot \nabla) h+\nabla \cdot \mathbf{q}=\sigma^{\epsilon}
$$

If the functions $h(T)$ depends only on $T$ then

$$
\rho C_{p} \frac{\partial}{\partial t} T+\rho C_{p}(\mathbf{v} \cdot \nabla) T-K_{\mathrm{eff}} \nabla^{2} T-\nabla K_{\mathrm{eff}} \cdot \nabla T=\sigma^{\epsilon}
$$

where $\frac{\partial h}{\partial T}=C_{p}$, with $C_{p}$ the specific heat at constant pressure, $\mathbf{q}=-K_{\text {eff }} \nabla T$, and $\nabla K_{\text {eff }}=$

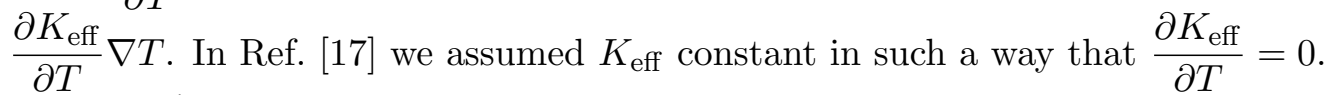

Let $\dot{Q}_{i}{ }^{\prime}$ be the sum of the dissipated heat of all the cylinders in column $i$ (i.e., $\dot{Q}_{i}{ }^{\prime}=\sum_{j}{\dot{Q_{i j}}}{ }^{\prime}$, being $\dot{Q_{i j}}{ }^{\prime}$ the heat dissipated by the $(i, j)$-nanodevice). In other words, the function $\sigma^{\epsilon}$ describing the source terms in equation (2.2) is composed of all these several cylinders in the column, i.e., it is a discretized term acting on the positions of the several cylinders.

If we assume $\mathbf{v}=(v, 0,0)$ and $\mathbf{q}=-K_{\text {eff }} \partial T / \partial x$ with $K_{\text {eff }}$ the constant value given by the reciprocal of Eq. (2.21) (or of expressions $(2.22)$ or $(2.23)$ ) and $\sigma^{\epsilon}=\dot{Q}_{i}{ }^{\prime}$, then the general steady-state solution of $(3.25)$ is

$$
T_{0}(x)=c_{1}+c_{2} \mathrm{e}^{\frac{\rho C_{p} v}{K_{\text {effo }}} x}+\frac{\sigma_{0}^{\epsilon}}{\rho C_{p} Q_{v}} x .
$$

where $c_{1}$ and $c_{2}$ are integration constants related to the temperature and temperature gradient at $x=0$. In Ref. [17] it was chosen $\sigma_{0}^{\epsilon}=\dot{Q}_{i}{ }^{\prime}$.

Now we assume that the source term $\sigma^{\epsilon}$ in Eq. (3.24) is given in average by

$$
\sigma^{\epsilon}=\frac{1}{A_{1}} \sum_{i j} u_{i j} \dot{n}_{i j},
$$

where $u_{i j}$ is the dissipated energy per proccessed bit (which may be defined as $u_{i j}=T s_{i j}^{\prime}$ ) and $\dot{n}_{i j}$ the local operation rate, with $A_{1}$ the total area of the system. In more local terms, $\sigma^{\epsilon}$ may be related to the heat dissipated by the cylinders in the local position being considered.

\subsection{Perturbations in the temperature of the source terms}

In this subsection we are interested to study the linear stability of the stationary solution of equation (3.25) in terms of the temperature $T$, the thermal conductivity $K_{\text {eff }}$ and the dissipated energy per proccessed bit $u_{i j}$ in $\sigma^{\epsilon}$. To this end, let's consider 


$$
\begin{aligned}
& T(x, t)=T_{0}(x)+\hat{T} \exp (\mathrm{i}(k x-\omega t)) \\
& \sigma^{\epsilon}(x, t)=\sigma_{0}^{\epsilon}+\hat{\sigma}^{\epsilon} \exp (\mathrm{i}(k x-\omega t)) \\
& K_{\text {eff }}(x, t)=K_{\text {eff } 0}+\hat{K}_{\text {eff }} \exp (\mathrm{i}(k x-\omega t))
\end{aligned}
$$

where $T_{0}(x), \sigma_{0}^{\epsilon}$ and $K_{\text {eff } 0}$ are the values of $T, \sigma^{\epsilon}$ and $K_{\text {eff }}$ in the steady state. In particular, $\sigma_{0}^{\epsilon}$ is given in Eq. (3.27). The coefficients $\hat{T}, \hat{\sigma}^{\epsilon}$ and $\hat{K}_{\text {eff }}$ are small perturbation of the fields, which we assume to depends only on $x$ and not on $y$ and $z$. Of course, this choice will give us the thresholds above which the flow is surely unstable, but nothing we can say about its stability, because the general case with the perturbations depending on $y$ and $z$ is lacking.

Let's recall the definition of stability for the stationary solution of our system.

Definition 3.1 The stationary solution $\Gamma_{0}=\left(T_{0}(x), \sigma_{0}^{\epsilon}, K_{\text {effo }}\right)$ is stable if $\forall \epsilon>0$ and fixed $t_{0}$ there exists $\eta=\eta(\epsilon, t)$ such that any solution $\Gamma(x, t)=\left(T(x, t), \sigma^{\epsilon}(x, t), K_{\text {eff }}(x, t)\right)$ with the condition that $\left|\Gamma\left(x, t_{0}\right)-\Gamma_{0}\right|<\eta$ implies that

$$
\left|\Gamma(x, t)-\Gamma_{0}\right|<\epsilon \quad \forall t>t_{0}
$$

REMARK 3.1 According to the above definition of stability, choosing $\forall \epsilon>0, t_{0}=0$ and any solution $\Gamma(x, t)=\left(T(x, t), \sigma^{\epsilon}(x, t), K_{\mathrm{eff}}(x, t)\right)$ with the expressions given by equations (3.28), (3.29) and (3.30), then we can write

$$
\left|\Gamma(x, t)-\Gamma_{0}\right|^{2}=\left|T(x, t)-T_{0}(x)\right|^{2}+\left|\sigma^{\epsilon}-\sigma_{0}^{\epsilon}\right|^{2}+\left|K_{\text {eff }}(x, t)-K_{\text {eff }}\right|^{2}=\left(\hat{T}^{2}+\hat{\sigma}^{2}+{\hat{K_{\text {eff }}}}^{2}\right) \mathrm{e}^{\omega_{\mathrm{i}} \mathrm{t}}
$$

Thus, choosing $\eta=\epsilon$ and $\omega_{i}<0$, then we have that $\left|\Gamma(x, 0)-\Gamma_{0}\right|=\sqrt{\left(\hat{T}^{2}+\hat{\sigma}^{2}+{\hat{K_{\mathrm{eff}}}}^{2}\right)}<$ $\eta$ implies that $\left|\Gamma(x, t)-\Gamma_{0}\right|=\sqrt{\left(\hat{T}^{2}+\hat{\sigma}^{2}+\hat{K}_{\text {eff }}^{2}\right)} \mathrm{e}^{\omega_{\mathrm{i}} / 2 \mathrm{t}}<\eta \mathrm{e}^{\omega_{\mathrm{i}} / 2 \mathrm{t}}<\eta=\epsilon$ because $\omega_{i}<0$.

In conclusion, we can assert that $\omega_{i} \geq 0$ is the condition for the instability of our stationary solution $\Gamma_{0}$ because $\mathrm{e}^{\omega_{\mathrm{i}} / 2 \mathrm{t}}$ becomes a growing function, such that solution $\Gamma(x, t)$ escapes from $\Gamma_{0}$. Of course, as stated above, we cannot conclude any about the stability of $\Gamma_{0}$ because we should prove that the stability condition is verified for any solution $\Gamma(x, y, z, t)$.

In this subsection it is assumed that

$$
\hat{\sigma^{\epsilon}}=\frac{1}{A} \sum_{i j} \hat{T} s_{i j} \dot{n}_{i j}
$$

namely, the perturbation lies in the dissipated energy. In the next subsection we will consider the perturbation of the local operation rate $\dot{n}_{i j}$.

After inserting Eq. (3.28) into equation (3.25) and using the stationary solution (3.26), we find:

$$
-\mathrm{i} \omega \rho C_{p} \hat{T}+\mathrm{i} k \rho C_{p} v \hat{T}+k^{2} \hat{T} K_{\mathrm{eff} 0}-\hat{K}_{\mathrm{eff}} \frac{\partial^{2} T_{0}}{\partial x^{2}}-\mathrm{i} k \hat{K}_{\mathrm{eff}} \frac{\partial T_{0}}{\partial x}=\frac{1}{A} \sum_{i j} \hat{T} s_{i j} \dot{n}_{i j}
$$


Since only the fourth and fifth terms in Eq. (3.34) depend on $x$, they must be zero, namely $-\hat{K}_{\text {eff }} \frac{\partial^{2} T_{0}}{\partial x^{2}}-\mathrm{i} k \hat{K}_{\text {eff }} \frac{\partial T_{0}}{\partial x}=0$, which occurs for $\hat{K}_{\text {eff }}=0$. Hence, equation (3.34) can be splitted into real and imaginary part:

$$
\begin{aligned}
& \left(\omega_{i} \rho C_{p}+k^{2} K_{\text {eff } 0}-\frac{1}{A} \sum_{i j} s_{i j} \dot{n}_{i j}\right) \hat{T}=0 \\
& \left(-\omega_{r}+k v\right) \rho C_{p} \hat{T}=0
\end{aligned}
$$

Then, in order that $\hat{T}$ may be different from zero, we find from (3.35) and (3.36)

$$
\omega_{i}=\frac{1}{\rho C_{p}}\left[-k^{2} K_{\mathrm{eff} 0}+\frac{1}{A} \sum_{i j} s_{i j} \dot{n}_{i j}\right]
$$

and

$$
\omega_{r}=k v
$$

Expression (3.38) yields the speed of propagation of the temperature signals, given by $v_{T}=\omega_{r} / k \equiv v$. According to our Remark 3.1, expression (3.37) shows the instability condition $\left(\omega_{i} \geq 0\right)$ in terms of the several quantities, namely

$$
\frac{1}{\rho C_{p}}\left[-k^{2} K_{\mathrm{eff} 0}+\frac{1}{A} \sum_{i j} s_{i j} \dot{n}_{i j}\right] \geq 0,
$$

which, since $\rho C_{p}>0$, becomes

$$
\frac{1}{A} \sum_{i j} s_{i j} \dot{n}_{i j} \geq k^{2} K_{\mathrm{eff} 0}
$$

REMARK 3.2 Expression (3.40) is the instability condition of the solution (3.26) and it is one of the main results of this paper, which shows the compromise of two central physical quantities, namely $\frac{\partial \sigma^{\epsilon}}{\partial T}=\frac{1}{A} \sum_{i j} s_{i j} \dot{n}_{i j}$ and $K_{\text {eff. }}$. The term $k^{2} K_{\text {eff refers to the rate of heat }}$ removal by conduction, $\frac{\partial \sigma^{\epsilon}}{\partial T}>0$ means that an increase in temperature will increase heat dissipation rate. When this latter effect increases faster than the rate of heat removal, the temperature perturbation will increase with time. Note that the most dangerous perturbations are those with $k$ smaller than $K_{\text {effo }}^{-1} \frac{\partial \sigma^{\epsilon}}{\partial T}$.

\subsection{Perturbations in the computing rate of the source terms}

Let's assume now variations in the computing rate $\dot{n}_{i j}$ of the source term $\sigma^{\epsilon}$ which are unavoidable in any computer, and which may depend both on time and on space. Since perturbations in the computing rate may span in a large range of possibilities, here we consider perturbations of the form in such a way we consider 


$$
\begin{aligned}
& T(x, t)=T_{0}(x)+\hat{T} \exp (\mathrm{i}(k x-\omega t)) \\
& \sigma^{\epsilon}(x, t)=\sigma_{0}^{\epsilon}+\hat{\sigma}^{\epsilon} \exp (\mathrm{i}(\bar{k} x-\bar{\omega} t) \\
& K_{\text {eff }}(x, t)=K_{\text {eff } 0}+\hat{K}_{\text {eff }} \exp (\mathrm{i}(k x-\omega t))
\end{aligned}
$$

with

$$
\hat{\sigma}^{\epsilon}=\frac{1}{A} \sum_{i j} T s_{i j} \hat{\dot{n}}
$$

with $\hat{\dot{n}}=\max \left\{n_{i j}, 1 \leq i \leq N\right.$ and $\left.1 \leq j \leq M\right\}, \bar{k} \in \mathcal{R}$ and $\bar{\omega}=\bar{\omega}_{r}+\mathrm{i} \bar{\omega}_{i} \in \mathcal{C}$ with $\bar{\omega}_{i}=$ $\max \left\{\omega_{i}^{i j}, 1 \leq i \leq N\right.$ and $\left.1 \leq j \leq M\right\}$. Let's assume also that $s_{i j}=\bar{s} \forall 1 \leq i \leq N$ an $\forall 1 \leq$ $j \leq M$.

Introducing (3.41), (3.42) and (3.43) in (3.25) one finds:

$$
-\mathrm{i} \omega \rho C_{p} \hat{T}+\mathrm{i} k \rho C_{p} v \hat{T}+k^{2} \hat{T} K_{\text {eff } 0}-\hat{K}_{\text {eff }} \frac{\partial^{2} T_{0}}{\partial x^{2}}-\mathrm{i} k \hat{K}_{\text {eff }} \frac{\partial T_{0}}{\partial x}=T_{0} \bar{s} \hat{\dot{n}} \mathrm{e}^{\mathrm{i}((\bar{k}-k) x-(\bar{\omega}-\omega) t)}
$$

which can be splitted into real and imaginary part

$$
\begin{aligned}
& \omega_{i} \rho C_{p} \hat{T}+k^{2} \hat{T} K_{\mathrm{eff} 0}-\hat{K}_{\mathrm{eff}} \frac{\partial^{2} T_{0}}{\partial x^{2}}=T_{0} \overline{\hat{s}} \hat{\dot{n}} \mathrm{e}^{\left(\bar{\omega}_{i}-\omega_{i}\right) t} \cos \left[\left((\bar{k}-k) x-\left(\bar{\omega}_{r}-\omega_{r}\right) t\right)\right] \\
& -\omega_{r} \rho C_{p} \hat{T}+k \rho C_{p} v \hat{T}-k \hat{K}_{\mathrm{eff}} \frac{\partial T_{0}}{\partial x}=T_{0} \bar{s} \hat{\dot{n}} \mathrm{e}^{\left(\bar{\omega}_{i}-\omega_{i}\right) t} \sin \left[\left((\bar{k}-k) x-\left(\bar{\omega}_{r}-\omega_{r}\right) t\right)\right]
\end{aligned}
$$

Since the unknowns in equations (3.46) and (3.47) are costants to be determined, then we need to remove the dependence of $x$ and $t$ from these equations. This means that

$$
\omega_{i}=\bar{\omega}_{i}, \quad \hat{K}_{\text {eff }}=0, \quad \omega_{r}=\bar{\omega}_{r} \quad k=\bar{k}
$$

Thus, equations (3.46) and (3.47) become

$$
\begin{gathered}
\left(\omega_{i} \rho C_{p}+k^{2} K_{\text {effo }}\right) \hat{T}=T_{0} \bar{s} \hat{\dot{n}} \\
\left(-\omega_{r}+k v\right) \rho C_{p} \hat{T}=0
\end{gathered}
$$

Equation (3.49) has the solution

$$
\hat{T}=\frac{T_{0} \bar{s} \hat{\dot{n}}}{\left(\bar{\omega}_{i} \rho C_{p}+\bar{k}^{2} K_{\text {eff } 0}\right)}
$$

while equation (3.50) has the solution $\omega_{r}=k v$.

The stability condition is the same we pointed out in the previous subsection, namely $\omega_{i}<0$. Thus, according to the results of the current subsection, we can assert that the stationary solution is unstable for $\omega_{i}=\bar{\omega}_{i} \geq 0$.

When the frequency $\bar{\omega}_{i}=0$, the condition for the instability is satisfied and the solution is unstable with the perturbation given by

$$
\hat{T}=\frac{T_{0} \bar{s} \hat{\dot{n}}}{\bar{k}^{2} K_{\text {eff } 0}}
$$


REMARK 3.3 If $\bar{\omega}_{i}<0$ (which would be stable), equations (3.51) and (3.52) place a stronger condition on the system, because it is needed that $\hat{T}+T_{0}<T_{\lambda}$, where $T_{\lambda}$ is the lambda transition temperature. If such temperature is reached, the refrigerating helium will be no longer superfluid. From here a restriction on the maximum value of $\hat{\dot{n}}$, the maximum local rate of variation of the computation rate, may be found; in particular, from equation (3.52) we find

$$
\frac{T_{0} \bar{s} \hat{\dot{n}}}{\left(\bar{\omega}_{i} \rho C_{p}+\bar{k}^{2} K_{\text {eff } 0}\right)}<T_{\lambda}-T_{0}
$$

\subsection{Perturbations by the external velocity}

Now, we study the linear stability of the solution (3.26) after perturbations due to the velocity $\mathbf{v}$, namely, if we assume

$$
\mathbf{v}=v \hat{\mathbf{x}}+\hat{\mathbf{v}}(x, y, z) \mathrm{e}^{\mathrm{i}(k x-\omega t)}
$$

with $\hat{\mathbf{v}}(x, y, z)=\left(\hat{v}_{x}(x, y, z), \hat{v}_{y}(x, y, z), \hat{v}_{z}(x, y, z)\right)$ and

$$
T=T_{0}(x)+\hat{T}(y, z) \mathrm{e}^{\mathrm{i}(k x-\omega t)} .
$$

with $k$ real and $\omega=\omega_{r}+\omega_{i}$.

Substituting Eq. (3.55) in Eq. (3.25), we obtain the following equation for $\hat{T}(x, y, z)$

$$
K_{\mathrm{eff}}\left(\frac{\partial^{2}}{\partial y^{2}}+\frac{\partial^{2}}{\partial z^{2}}\right) \hat{T}(y, z)-\left[\mathrm{i} \rho C_{p}(v k-\omega)+k^{2} K_{\mathrm{eff}}\right] \hat{T}(y, z)=\rho C_{p} \hat{v}_{x}(x, y, z) \frac{\partial}{\partial x} T_{0}
$$

Since only the right-hand side of Eq. (3.56) depends on $x$, then it must be equal to a regular function $f(y, z)$, namely $\rho C_{p} \hat{v}_{x}(x, y, z) \frac{\partial}{\partial x} T_{0}:=f(y, z)$. This implies that

$$
\hat{v}_{x}(x, y, z)=\frac{f(y, z)}{\rho C_{p} \frac{\partial}{\partial x} T_{0}}=\frac{f(y, z)}{\rho C_{p}\left(c_{2} \frac{\rho C_{p} v}{K_{\text {effo }}} \mathrm{e}^{\frac{\rho C_{p} v}{K_{\text {effo }}} x}+\frac{\sigma_{0}^{\epsilon}}{\rho C_{p} Q_{v}}\right)}
$$

By setting the imaginary terms in equation (3.56) zero then we find the dispersion relation $\omega_{r}=v k$, and equation (3.56) becomes

$$
K_{\mathrm{eff}}\left(\frac{\partial^{2}}{\partial y^{2}}+\frac{\partial^{2}}{\partial z^{2}}\right) \hat{T}(y, z)-\left[\rho C_{p} \omega_{i}+k^{2} K_{\mathrm{eff}}\right] \hat{T}(y, z)=f(y, z) .
$$

Equation (3.58) is the Poisson equation which, with regular boundary conditions,, admits solution if the function $f(y, z)$ is regular .

In conclusion, the stationary solution (3.26) is linear stable whenever $\omega_{i}<0$ and $R e_{q}<$ $R e_{q}^{c}$

\section{Conclusions}

In this paper we have explored a concrete relation between two different topics: thermodynamics of computation, and computer refrigeration. Thermodynamics of computation focuses the attention on dissipation and heating related to computation. For the refrigeration, we have 
considered the particular case of refrigeration with supefluid helium, because we have in mind computers at very low temperature, as motivated by the interest in quantum computation.

We assumed that the refrigeration of the particular simplified model of computer considered here (assumed as a simple regular array of cylindrical chips [15], [16]) is given by equation (3.25). In that equation, $C_{p}$ is the specific heat of superfluid helium and $K_{\text {eff }}$ is the effective thermal conductivity, which is the reciprocal of the thermal resistivity (2.21) (or of its particular limiting expressions (2.22) and (2.23). Note that $C_{p}$ depends only on the material, whereas $K_{\text {eff }}$ depends also on the geometry of the channel in a complicated form, as we are dealing not with Fourier heat transport but whith phonon hydrodynamic heat transport.

In the right-hand side of equation (3.25), instead, there is the heating due to computation. This heating term is represented through expression (3.27), which is a central quantity in thermodynamics of computation, as in it there appear the computation rate $(\dot{n})$ and the entropy produced per each bit being processed $\left(s_{i j}^{\prime}\right)$.

It is found that at constant computation rate, i.e., if the only perturbations to the heating rate come from temperature perturbations (and their feedback consequences of the heating rate), the linear stability condition is given by (3.40), i.e. it depends on the rate of variation of the heating rate $\sigma^{\epsilon}$, with temperature variations depending on the modulus of the wavevector $k$ of the perturbation, and on the expression for the effective termal conductivity $K_{\text {effo }}$. This expression is of particular physical interest as it relates two quantities coming from the different fields of superfluid helium heat transport $\left(K_{\text {eff }}\right)$ and from thermodynamics of computation $\left(\sigma^{\epsilon}\right)$.

The second main results are Eqs. (3.48) and (3.51) expressing the condition that $\bar{\omega}_{i} \geq 0$ and the relation between the amplitudes of computation rate perturbation $\hat{\dot{n}}$ and of temperature perturbation $\hat{T}$. From equation (3.51) itself no explicit conclusion follows. The conclusion arises when we impose that the total temperature of the system (namely $T_{0}+\hat{T}$ as the maximum temperature) should not exceed the lambda temperature $T_{\lambda}$ characterising the superfluidnormal fluid phase transition. In this case the maximum admisible value for the rate of change of variation of the local conputation rate per cylinder is restricted by (3.53). It is worth to mention that for temperature next to the transition temperature $T_{\lambda}$, the mathematical model of superfluid helium (2.1)-(2.4) has to be extended with an equation for the order parameter in order to describe the phase transition between He I (classical fluid) to He II (superfluid helium) [32], [33]). This will be the aims of our future studies.

Equation (3.51) is not related to an indefinite increase of the local temperature, but to a finite (maybe small) increase, leading the superfluid helium out of the superfluidity regime, in which case its heat transport ability decreases substantially.

The result in Eq. (3.45) has been obtained in the particular situation in which the perturbations in the local computing rate are given by waves with the same wavevector and frequency than temperature perturbations. More general situations may take place, leading to other particular results, but Eq. (3.51) is of sufficient interest because it illustrates the restriction on the stability of the steady-state temperature profile when computation rate is varied. In a more generally setting, the array of nanocylinders would not be exactly periodic, and the effects of inhomogeneities in the density of nanocylinders per unit area should also be considered.

A more general discussion on the stability of the whole system (2.1)-(2.4) will be the aim for future studies as well as the discussion for the boundary conditions. 


\section{Acknowledgements}

This work was supported by National Group of Mathematical Physics (GNFM-INdAM). LG is supported by the Engineering and Physical Sciences Research Councile (EPSRC), grant n. EP/R005192/1. D.J. acknowledges the financial support from the Dirección General de Investigación of the Spanish Ministry of Economy and Competitiveness under grant TEC201567462-C2-2-R and of the Direcció General de Recerca of the Generalitat of Catalonia, under grant 2017 SGR-1018 and to Consolider Program Nanotherm (grant CSD-2010-00044) of the Spanish ministry of Science and Innovation. The authors would thank the anonymous referee for his help to improve our paper.

\section{References}

[1] C. H. Bennett, "The thermodynamics of computation: a review," International Journal of Theoretical Physics, vol. 21, no. 12, pp. 905-940, 1982.

[2] H. S. Leff and A. F. Rex, Maxwell's demon: entropy, information, computing. Princeton University Press, 2014.

[3] W. H. Zurek, "Algorithmic randomness and physical entropy," Physical Review A, vol. 40, no. 8 , p. $4731,1989$.

[4] H. J. Caulfield and L. Qian, "Thermodynamics of computation," in Encyclopedia of Complexity and Systems Science, pp. 9127-9137, Springer, 2009.

[5] T. Iwayama, M. Sueyoshi, and T. Watanabe, "Linear stability analysis of parallel shear flows for an inviscid generalized two-dimensional fluid system," Journal of Physics A: Mathematical and Theoretical, vol. 46, no. 6, p. 065501, 2013.

[6] H. Kim, S. Kwon, J. C. Padrino, and T. Funada, "Viscous potential flow analysis of capillary instability with heat and mass transfer," Journal of Physics A: Mathematical and Theoretical, vol. 41, no. 33, p. 335205, 2008.

[7] A. E. Hassanien, M. Elhoseny, and J. Kacprzyk, Quantum Computing: An Environment for Intelligent Large Scale Real Application. Springer, 2018.

[8] M. Lanzagorta and J. Uhlmann, "Quantum computer science," Synthesis Lectures on Quantum Computing, vol. 1, pp. 1-124, 2008.

[9] B. Ruggiero, P. Delsing, C. Granata, Y. A. Pashkin, and P. Silvestrini, Quantum computing in solid state systems. Springer Science \& Business Media, 2006.

[10] K. Y. Tan, M. Partanen, R. E. Lake, J. Govenius, S. Masuda, and M. Möttönen, "Quantum-circuit refrigerator," Nature Communications, vol. 8, p. 15189, 2017.

[11] S. W. Van Sciver, Helium cryogenics. Berlin: Springer, second ed., 2012.

[12] G. Pilbratt, J. Riedinger, T. Passvogel, G. Crone, D. Doyle, U. Gageur, A. Heras, C. Jewell, L. Metcalfe, S. Ott, et al., "Herschel space observatory-an esa facility for far-infrared and submillimetre astronomy," Astronomy 8 Astrophysics, vol. 518, p. L1, 2010. 
[13] P. Lebrun, "Superfluid helium cryogenics for the large hadron collider project at cern," Cryogenics, vol. 34, pp. 1-8, 1994.

[14] J. Casas, A. Cyvoct, P. Lebrun, M. Marquet, L. Tavian, and R. van Weelderen, "Design concept and first experimental validation of the superfluid helium system for the large hadron collider (lhc) project at cern," Cryogenics, vol. 32, pp. 118-121, 1992.

[15] D. Jou, M. Sciacca, A. Sellitto, and L. Galantucci, "Refrigeration of an array of cylindrical nanosystems by superfluid helium counterflow," Accademia Peloritana dei Pericolanti Classe FF. MM. NN.

[16] M. Sciacca, A. Sellitto, L. Galantucci, and D. Jou, "Refrigeration of an array of cylindrical nanosystems by superfluid helium counterflow," International Journal of Heat and Mass Transfer, vol. 104, pp. 584-594, 2017.

[17] D. Jou, L. Galantucci, and M. Sciacca, "Refrigeration of an array of cylindrical nanosystems by flowing superfluid helium," Journal of Low Temperature Physics, vol. 187, no. 5-6, pp. 602-610, 2017.

[18] B. Lambson, D. Carlton, and J. Bokor, "Exploring the thermodynamic limits of computation in integrated systems: Magnetic memory, nanomagnetic logic, and the Landauer limit," Physical Review letters, vol. 107, no. 1, p. 010604, 2011.

[19] W. Porod, R. Grondin, D. Ferry, and G. Porod, "Dissipation in computation," Physical Review Letters, vol. 52, no. 3, p. 232, 1984.

[20] R. Landauer, "Dissipation in computation," Physical Review Letters, vol. 53, no. 12, p. $1205,1984$.

[21] D. Jou, G. Lebon, and M. Mongiovì, "Second sound, superfluid turbulence, and intermittent effects in liquid helium II," Physical Review B, vol. 66, p. 224509 (9 pages), 2002.

[22] D. Jou and M. Mongiovì, "Description and evolution of anisotropy in superfluid vortex tangles with counterflow and rotation," Physical Review B, vol. 74, p. 054509 (11 pages), 2006 .

[23] M. Mongiovì, "Extended irreversible thermodynamics of liquid helium II," Physical Review B, vol. 48, pp. 6276-6283, 1993.

[24] M. Sciacca, D. Jou, and M. S. Mongiovì, "Effective thermal conductivity of helium II: from Landau to Gorter-Mellink regimes," Zeitschrift für angewandte Mathematik und Physik, vol. 66, pp. 1835-1851, 2015.

[25] M. S. Mongiovì, D. Jou, and M. Sciacca, "Non-equilibrium thermodynamics, heat transport and thermal waves in laminar and turbulent superfluid helium," Physics Reports, vol. 726 , pp. 1-71, 2018.

[26] M. Sciacca and L. Galantucci, "Effective thermal conductivity of superfluid helium: laminar, turbulent and ballistic regimes," Communications in Applied and Industrial Mathematics, vol. 7, pp. 111-129, 2016. 
[27] L. Saluto, M. S. Mongioví, and D. Jou, "Longitudinal counterflow in turbulent liquid helium: velocity profile of the normal component," Zeitschrift für angewandte Mathematik und Physik, vol. 65, pp. 531-548, 2014.

[28] L. Saluto, D. Jou, and M. Mongiovì, "Contribution of the normal component to the thermal resistance of turbulent liquid helium," Zeitschrift für angewandte Mathematik und Physik, vol. 66, no. 4, pp. 1853-1870, 2015.

[29] M. Sciacca, A. Sellitto, and D. Jou, "Transition to ballistic regime for heat transport in helium II," Physics Letters A, vol. 378, pp. 2471-2477, 2014.

[30] T. Tatsumi and T. Yoshimura, "Stability of the laminar flow in a rectangular duct," Journal of Fluid Mechanics, vol. 212, pp. 437-449, 1990.

[31] D. Jou and M. Sciacca, "Quantum Reynolds number for superfluid counterflow turbulence," in Bollettino di Matematica Pura e Applicata (M. S. Mongiovì, M. Sciacca, and S. Triolo, eds.), vol. VI, pp. 95-103, Aracne editrice, 2013.

[32] M. Mongiovì and L. Saluto, "Effects of heat flux on $\lambda$-transition in liquid 4He," Meccanica, vol. 49, pp. 2125-2137, 2014.

[33] L. Ardizzone, M. S. Mongiovì, and L. Saluto, "Non-equilibrium thermodynamical description of superfluid transition in liquid helium," Journal of Non-Equilibrium Thermodynamics, vol. 42, no. 4, pp. 371-385, 2017. 